BULLETIN (New Series) OF THE

AMERICAN MATHEMATICAL SOCIETY

Volume 38, Number 3, Pages 273-291

S 0273-0979(01)00903-X

Article electronically published on March 27, 2001

\title{
FRAMES, RIESZ BASES, AND DISCRETE GABOR/WAVELET EXPANSIONS
}

\author{
OLE CHRISTENSEN
}

\begin{abstract}
This paper is a survey of research in discrete expansions over the last 10 years, mainly of functions in $L^{2}(\mathbb{R})$. The concept of an orthonormal basis $\left\{f_{n}\right\}$, allowing every function $f \in L^{2}(\mathbb{R})$ to be written $f=\sum c_{n} f_{n}$ for suitable coefficients $\left\{c_{n}\right\}$, is well understood. In separable Hilbert spaces, a generalization known as frames exists, which still allows such a representation. However, the coefficients $\left\{c_{n}\right\}$ are not necessarily unique. We discuss the relationship between frames and Riesz bases, a subject where several new results have been proved over the last 10 years. Another central topic is the study of frames with additional structure, most important Gabor frames (consisting of modulated and translated versions of a single function) and wavelets (translated and dilated versions of one function). Along the way, we discuss some possible directions for future research.
\end{abstract}

\section{INTRODUCTION}

The theory for frames and bases has developed very fast over the last 10 years, especially in the context of wavelets and Gabor systems. For a whole generation of researchers and Ph.D. students, the starting point has been the article 24] by Daubechies, her book [25, and the survey paper by Heil and Walnut 37]. The purpose of the present paper is to give a unified approach to the newest research generalizing the results from [37]. With a few exceptions we use the notation from [37. Thus, the paper can be thought of as an (independent) continuation of 37.

Let $L^{2}(\mathbb{R})$ denote the space of square integrable functions (w.r.t. Lebesgue measure). We usually think about functions $f \in L^{2}(\mathbb{R})$ as signals. Recall that a basis $\left\{f_{n}\right\}$ for $L^{2}(\mathbb{R})$ allows every $f \in L^{2}(\mathbb{R})$ to be written

$$
f=\sum_{n} c_{n} f_{n}
$$

for a unique set of scalar coefficients $\left\{c_{n}\right\}$. Usually we think about (11) as a decomposition of the "complicated" signal $f$ into a sum of the "simpler building blocks" $f_{n}$ that might be easier to work with. For instance, if $T$ is a bounded operator on $L^{2}(\mathbb{R})$ and the action on the building blocks $f_{n}$ are known, we can find the action on an arbitrary signal $f \in L^{2}(\mathbb{R})$ as $T f=\sum_{n} c_{n} T f_{n}$.

Received by the editors July 25, 2000.

2000 Mathematics Subject Classification. Primary 41A58, 42C15.

Key words and phrases. Frames, Riesz bases, discrete expansions, Gabor systems, wavelets, frames of exponentials. 
Applications might ask for bases with special properties that make them easier to deal with. For instance, $\left\{f_{n}\right\}$ is an unconditional basis if (1) converges unconditionally for each $f$, i.e., if every permutation $\sigma(n)$ of the index set makes $\sum_{\sigma(n)} c_{n} f_{n}$ converge to $f$. This happens for example if $\left\{f_{n}\right\}$ is an orthonormal basis or, more generally, a Riesz basis (defined in Section 2.3).

The last 10 years have shown that it very often is convenient to work with frames instead of bases: if $\left\{f_{n}\right\}$ is a frame for $L^{2}(\mathbb{R})$, each $f \in L^{2}(\mathbb{R})$ can still be represented via an unconditionally convergent series as in (10), but usually the set of coefficients $\left\{c_{n}\right\}$ is not unique. This has several advantages: the lack of uniqueness opens up the possibility of choosing the coefficients that fit a certain application best, and it also makes the representation (1) of a signal $f$ less sensitive to noise. Also, since the (orthonormal) basis condition is very strong, it might be difficult to find a basis satisfying extra conditions that a certain application requires. The frame condition is weaker, and therefore one can often find a frame enjoying special properties which are impossible for a basis. We mention some important examples in connection with Gabor systems and wavelets in Sections 4.2 and 5 .

Frames were originally defined by Duffin and Schaefer in the early fifties, in the context of nonharmonic Fourier Series (see Section 3). The interested reader might be surprised by seeing how much of the frame theory was developed already in the first paper [28]! The breakthrough in frames came in 1986, when Grossmann [26] observed the relationship to wavelets.

For computational purposes, it is essential to consider frames (or bases) with a simple structure. This is the motivation behind Gabor frames and wavelets. A Gabor frame consists of time-frequency shifts of a single function $g \in L^{2}(\mathbb{R})$; i.e., it has the form $\left\{e^{i 2 \pi m b x} g(x-n a)\right\}_{m, n \in \mathbb{Z}}$ for some parameters $a, b>0$. Similarly, a wavelet frame $\left\{\frac{1}{a^{j / 2}} \psi\left(\frac{x}{a^{j}}-k b\right)\right\}_{j, k \in \mathbb{Z}}$ consists of shifted and scaled versions of the single function $\psi \in L^{2}(\mathbb{R})$. Wavelets have been studied intensively the last 10 years, since the breakthrough of multiresolution analysis made it possible to construct orthonormal bases of wavelets with very desirable properties. Since multiresolution analysis is already well presented in books and monographs, we shall not discuss it here. However, we include a short description of a generalization called frame multiresolution analysis.

The paper is organized as follows. We begin in Section 2 with the theory for frames and Riesz bases in general Hilbert spaces. An equivalent characterization of frames is given, and the relationship between frames and Riesz bases is discussed in detail.

Section 3 deals with frames of exponentials in $L^{2}(-\pi, \pi)$. The section is mainly included as background for the study of Gabor frames, which is the subject of Section 4. Sufficient conditions (generalizing those in [37]) for $\left\{e^{i 2 \pi m b x} g(x-n a)\right\}_{m, n \in \mathbb{Z}}$ to be a frame are presented. We discuss the Balian-Low Theorem, which is a negative result in Gabor-frame theory: loosely speaking, it says that if $\left\{e^{i 2 \pi m b x} g(x-n a)\right\}_{m, n \in \mathbb{Z}}$ is a Riesz basis, then $g$ cannot be well localized in both time and frequency. As we shall see, this problem can be circumvented by considering Wilson bases. The section ends with a discussion of open problems related to irregular Gabor systems.

Section 5 is devoted to wavelets. A sufficient condition for $\left\{\frac{1}{a^{j / 2}} \psi\left(\frac{x}{a^{j}}-k b\right)\right\}_{m, n \in \mathbb{Z}}$ to be a frame is presented, and a short discussion of frame multiresolution analysis is given. 


\section{Frames AND BASES in Hilbert spaCES}

Let $\mathcal{H}$ be a separable Hilbert space with the inner product $\langle\cdot, \cdot\rangle$ linear in the first entry. The purpose of this section is to present some recent results about frames and bases in general Hilbert spaces. No special structure is assumed at the moment, in contrast to the following sections.

For convenience we don't specify the index set when we consider a family of elements $\left\{f_{n}\right\} \subseteq \mathcal{H}$. We always assume the index set to be countable. When we speak about a series $\sum c_{n} f_{n}$, it is understood that the convergence is with respect to a certain enumeration, which is chosen once for all (but it will become clear in the next subsection that we usually obtain unconditionally convergent series).

2.1. Bessel sequences. As we shall see in Section 2.2, the frame condition can naturally be split into an upper frame condition and a lower frame condition. Frequently, it is convenient to look at those conditions separately. A sequence satisfying the upper frame condition is called a Bessel sequence:

Definition 2.1. A family of elements $\left\{f_{n}\right\} \subseteq \mathcal{H}$ is called a Bessel sequence if there exists a constant $B>0$ such that

$$
\sum\left|<f, f_{n}>\right|^{2} \leq B\|f\|^{2}, \forall f \in \mathcal{H} .
$$

Bessel sequences can be characterized in terms of the so-called pre-frame operator, introduced in the lemma below.

Lemma 2.2. $\left\{f_{n}\right\}$ is a Bessel sequence if and only if

$$
T:\left\{c_{n}\right\} \rightarrow \sum c_{n} f_{n}
$$

is a well defined operator from $\ell^{2}$ into $\mathcal{H}$. In that case $T$ is automatically bounded, and the adjoint of $T$ is given by

$$
T^{*}: \mathcal{H} \rightarrow \ell^{2}, \quad T^{*} f=\left\{<f, f_{n}>\right\} .
$$

In the literature, Lemma 2.2 is usually formulated as " $\left\{f_{n}\right\}$ is a Bessel sequence $\Leftrightarrow T$ is bounded". However, it follows by the Banach-Steinhaus theorem that $T$ is automatically bounded if $T$ is well defined on $\ell^{2}$. As an important consequence of Lemma 2.2 we notice that if $\left\{f_{n}\right\}$ is a Bessel sequence, then $\sum c_{n} f_{n}$ converges unconditionally for all $\left\{c_{n}\right\} \in \ell^{2}$.

2.2. Frames - equivalent characterizations. A frame can be thought of as some kind of "generalized basis". In this section we present the formal definition and some equivalent characterizations.

Definition 2.3. A family of elements $\left\{f_{n}\right\} \subseteq \mathcal{H}$ is called a frame for $\mathcal{H}$ if there exist constants $A, B>0$ such that

$$
A\|f\|^{2} \leq \sum\left|<f, f_{n}>\right|^{2} \leq B\|f\|^{2}, \quad \forall f \in \mathcal{H} .
$$

Any numbers $A, B$ for which (4) is valid are called frame bounds. They are not unique. The optimal frame bounds are the biggest possible value for $A$ and the smallest possible value for $B$ in (44). If we can choose $A=B$, the frame is called tight. If a frame ceases to be a frame when an arbitrary element is removed, the frame is said to be exact. 
When $\left\{f_{n}\right\}$ is a frame, the pre-frame operator $T$ from Section 2.1 is well defined; by composing $T$ with its adjoint $T^{*}$ we obtain the frame operator

$$
S: \mathcal{H} \rightarrow \mathcal{H}, \quad S f=T T^{*} f=\sum<f, f_{n}>f_{n}
$$

It is not difficult to prove that $S$ is bounded, positive and surjective; see e.g. [37. This leads to the frame decomposition:

Theorem 2.4. Given a frame $\left\{f_{n}\right\}$ with frame operator $S$, every $f \in \mathcal{H}$ can be represented as

$$
f=S S^{-1} f=\sum<f, S^{-1} f_{n}>f_{n} .
$$

Thus, a frame $\left\{f_{n}\right\}$ allows every $f \in \mathcal{H}$ to be written as a superposition of the frame elements. This property is very similar to the property (1) for a basis! The main difference is that for a frame $\left\{f_{n}\right\}$, the coefficients $<f, S^{-1} f_{n}>$ in (6) generally can be replaced by other coefficients. For more details we refer to the discussion following Theorem 2.8 below.

By Parseval's equality, an orthonormal basis $\left\{e_{n}\right\}$ is a frame with $A=B=1$. By adding a finite sequence or, more generally, a Bessel sequence $\left\{g_{n}\right\}$, we obtain a frame $\left\{e_{n}\right\} \cup\left\{g_{n}\right\}$. In finite dimensional spaces, every frame is the union of a basis and some "remaining vectors". This is not the case in infinite dimensional spaces; cf. the discussion after Theorem 2.8

Starting with an orthonormal basis $\left\{e_{n}\right\}_{n=1}^{\infty}$, many more or less artificial frames can be constructed. For example, the family

$$
\left\{e_{1}, \frac{1}{\sqrt{2}} e_{2}, \frac{1}{\sqrt{2}} e_{2}, \frac{1}{\sqrt{3}} e_{3}, \frac{1}{\sqrt{3}} e_{3}, \frac{1}{\sqrt{3}} e_{3}, \cdots\right\}
$$

will also be a frame with bounds $A=B=1$. We shall not discuss any such example, but refer to the following sections, where frames important for applications are presented.

The frame condition can be expressed entirely in terms of properties of the operator $T$. Theorem 2.5 below is a slight generalization of a result from [13] with a new elementary proof.

Theorem 2.5. A sequence $\left\{f_{n}\right\} \subseteq \mathcal{H}$ is a frame for $\mathcal{H}$ if and only if the mapping

$$
T:\left\{c_{n}\right\} \rightarrow \sum c_{n} f_{n}
$$

is a well defined mapping of $\ell^{2}$ onto $\mathcal{H}$.

Proof. First, suppose that $\left\{f_{n}\right\}$ is a frame. Since $\left\{f_{n}\right\}$ is a Bessel sequence, $T$ is a bounded operator from $\ell^{2}$ into $\mathcal{H}$ by Lemma 2.2. By (6), the frame operator $S=T T^{*}$ is surjective. Thus $T$ is surjective.

Now suppose that $T$ is a well defined operator from $\ell^{2}$ onto $\mathcal{H}$. By Lemma $2.2\left\{f_{n}\right\}$ satisfies the upper frame condition. Consider the restriction of $T$ to an operator on the orthogonal complement of the kernel of $T$; i.e., let $\tilde{T}:=T_{N_{T}}$ : $N_{T}^{\perp} \rightarrow \mathcal{H}$. Clearly $\tilde{T}$ is a bounded, bijective linear mapping, so it has a bounded inverse $T^{\dagger}:=\tilde{T}^{-1}: \mathcal{H} \rightarrow N_{T}^{\perp}$. By writing $T^{\dagger} f=\left\{\left(T^{\dagger} f\right)_{n}\right\}$ for $f \in \mathcal{H}$, we have

$$
f=T T^{\dagger} f=\sum\left(T^{\dagger} f\right)_{n} f_{n} .
$$


Thus

$$
\begin{aligned}
& \|f\|^{4}=<f, f>^{2}=\left|<\sum\left(T^{\dagger} f\right)_{n} f_{n}, f>\right|^{2} \\
\leq & \sum\left|\left(T^{\dagger} f\right)_{n}\right|^{2} \cdot \sum\left|<f, f_{n}>\right|^{2} \leq\left\|T^{\dagger}\right\|^{2} \cdot\|f\|^{2} \sum\left|<f, f_{n}>\right|^{2} ;
\end{aligned}
$$

we conclude that

$$
\sum\left|<f, f_{n}>\right|^{2} \geq \frac{1}{\left\|T^{\dagger}\right\|^{2}}\|f\|^{2}, \forall f \in \mathcal{H}
$$

The operator $T^{\dagger}$ constructed in the proof of Theorem [2.5] is usually called the pseudo-inverse of $T$. As the proof shows, $\frac{1}{\left\|T^{\dagger}\right\|^{2}}$ is a lower frame bound for $\left\{f_{n}\right\}$. Actually, this is the optimal lower bound. In [13] it is proved that the optimal bounds for a frame are given by

$$
A=\frac{1}{\left\|T^{\dagger}\right\|^{2}}=\frac{1}{\left\|S^{-1}\right\|}, \quad B=\|T\|^{2}=\|S\| .
$$

In Proposition 4.5 below, we use Theorem 2.5 to prove that a certain family constitutes a frame. Sometimes Theorem [2.5 can also be used to conclude that $\left\{f_{n}\right\}$ is not a frame:

Example 2.6. Let $\left\{e_{n}\right\}_{n=1}^{\infty}$ be an orthonormal basis for $\mathcal{H}$ and define

$$
f_{n}:=e_{n}+e_{n+1}, n \in \mathbb{N}
$$

Then $\left\{f_{n}\right\}_{n=1}^{\infty}$ is a Bessel sequence, but $e_{1}$ cannot be written $e_{1}=\sum_{n=1}^{\infty} c_{n} f_{n}$ for any sequence $\left\{c_{n}\right\}_{n=1}^{\infty} \subseteq \ell^{2}(\mathbb{N})$. Thus, by Theorem $2.5\left\{f_{n}\right\}_{n=1}^{\infty}$ is not a frame, despite the fact that $\mathcal{H}=\overline{\operatorname{span}}\left\{f_{n}\right\}_{n=1}^{\infty}$.

2.3. Riesz bases and frames. A family $\left\{f_{n}\right\}$ is a Riesz basis for $\mathcal{H}$ (or bounded unconditional basis in [37]) if there is a bounded invertible operator $U: \mathcal{H} \rightarrow \mathcal{H}$ and an orthonormal basis $\left\{e_{n}\right\}$ for $\mathcal{H}$ such that $f_{n}=U e_{n}$. By [51], p. $32,\left\{f_{n}\right\}$ is a Riesz basis if and only if $\left\{f_{n}\right\}$ is complete in $\mathcal{H}$ and there exist constants $A, B>0$ such that

$$
A \sum\left|c_{n}\right|^{2} \leq\left\|\sum c_{n} f_{n}\right\|^{2} \leq B \sum\left|c_{n}\right|^{2},
$$

for all finite sequences of scalars $\left\{c_{n}\right\}$.

It is illustrative to look at the relationship between Riesz bases and frames. To do so, we need the concept of biorthogonal sequences. Two sequences $\left\{f_{n}\right\}$ and $\left\{g_{n}\right\}$ in $\mathcal{H}$ are said to be biorthogonal if

$$
<f_{n}, g_{m}>=\delta_{n m}=\left\{\begin{array}{lll}
1 & \text { for } & n=m \\
0 & \text { for } & n \neq m
\end{array}\right.
$$

We refer to [51], p. 29, for the following elementary result:

Lemma 2.7. $\left\{f_{n}\right\}$ has a biorthogonal sequence if and only if it is minimal, meaning that, for all $m, f_{m} \notin \overline{\operatorname{span}}\left\{f_{n}\right\}_{n \neq m}$. Also, if a biorthogonal sequence exists, it is unique if and only if $\left\{f_{n}\right\}$ is complete.

In order to understand the importance of the concept, suppose that $\left\{f_{n}\right\}$ is a basis for $\mathcal{H}$ having a biorthogonal sequence $\left\{g_{n}\right\}$. Now we can easily find the 
representation of an arbitrary element $f \in \mathcal{H}$ in terms of the basis $\left\{f_{n}\right\}$ : with $f=\sum c_{n} f_{n}$, we have $<f, g_{m}>=\sum c_{n}<f_{n}, g_{m}>=c_{m}$; that is,

$$
f=\sum<f, g_{n}>f_{n}
$$

So when a biorthogonal sequence is known, we obtain a representation that is very similar to the well known representation using an orthonormal basis. It is easy to see that the biorthogonal sequence $\left\{g_{n}\right\}$ is also a basis.

We now turn to the important question about the relationship between frames and Riesz bases. Equivalent conditions for a frame to be a Riesz basis are collected in 41]:

Theorem 2.8. Let $\left\{f_{n}\right\}$ be a frame. Then the following are equivalent.

(i) $\left\{f_{n}\right\}$ is a Riesz basis for $\mathcal{H}$.

(ii) $\left\{f_{n}\right\}$ is an exact frame.

(iii) $\left\{f_{n}\right\}$ is minimal.

(iv) $\left\{f_{n}\right\}$ has a unique biorthogonal sequence.

(v) $\left\{S^{-1} f_{n}\right\}$ is a biorthogonal sequence.

(vi) If $\sum c_{n} f_{n}=0$ in $\mathcal{H}$ for a sequence of scalars $\left\{c_{n}\right\} \in \ell^{2}$, then $c_{n}=0$ for every $n$.

The implications (i) $\Leftrightarrow$ (ii) $\Leftrightarrow$ (iii) $\Leftrightarrow$ (iv) $\Leftrightarrow(\mathrm{v})$ are classical (see 37, Th. 2.2.2, Cor. 2.1.7.), while (i) $\Leftrightarrow$ (vi) appeared in 9]. As a consequence of Theorem 2.8 we note that if $\left\{f_{n}\right\}$ is a frame but not a Riesz basis, there exist non-zero sequences $\left\{c_{n}\right\} \in \ell^{2}$ such that $\sum c_{n} f_{n}=0$. Therefore $f \in L^{2}(\mathbb{R})$ can be written

$$
\begin{aligned}
f & =\sum<f, S^{-1} f_{n}>f_{n}+\sum c_{n} f_{n} \\
& =\sum\left(<f, S^{-1} f_{n}>+c_{n}\right) f_{n},
\end{aligned}
$$

showing that $f$ has many representations as superpositions of the frame elements.

Given a frame $\left\{f_{n}\right\}$, the family $\left\{S^{-1} f_{n}\right\}$ is also a frame, usually called the canonical dual; cf. [37]. In [42, Li gives a characterization of all duals, i.e., all Bessel sequences $\left\{g_{n}\right\}$ for which

$$
f=\sum<f, g_{n}>f_{n}, \quad \forall f \in \mathcal{H} .
$$

It is not hard to prove that for a Riesz basis the possible values for $A, B$ in (7) coincide with the frame bounds. In light of Theorem 2.8 it is natural to think about frames as "overcomplete bases", or, "families containing more elements than needed to be bases". Usually this intuitive way of looking at frames works well, but in a strict mathematical sense one needs to be careful: there exist frames $\left\{f_{n}\right\}_{n=1}^{\infty}$ for which no subfamily $\left\{f_{n}\right\}_{n \in I}, I \subseteq \mathbb{N}$, is a Riesz basis; cf. the frame $\left\{e_{1}, \frac{1}{\sqrt{2}} e_{2}, \frac{1}{\sqrt{2}} e_{2}, \frac{1}{\sqrt{3}} e_{3}, \frac{1}{\sqrt{3}} e_{3}, \frac{1}{\sqrt{3}} e_{3}, \cdots\right\}$ mentioned in Section 2.2. There even exist frames $\left\{f_{n}\right\}$ with $\left\|f_{n}\right\|=1, \forall n$, not even containing a Schauder basis! We refer to 9], 10 for the construction of such a frame. For a frame $\left\{f_{i}\right\}_{i \in I}$ with the property that every subfamily $\left\{f_{i}\right\}_{i \in J}, J \subseteq I$, is a frame for its closed span, with a common lower frame bound for all those frames, it can be proved that $\left\{f_{i}\right\}_{i \in I}$ contains a Riesz basis; cf. [18]. Frames with this property are called Riesz frames.

The condition (vi) in Theorem 2.8 (frequently called $\omega$-independence) is stronger than just linear independence. To illustrate that point, we state some more equivalent characterizations of Riesz bases. For notational convenience, we consider a frame $\left\{f_{n}\right\}_{n=1}^{\infty}$. For $N \in \mathbb{N}$, the finite subfamily $\left\{f_{n}\right\}_{n=1}^{N}$ is automatically a frame 
for the finite dimensional space $\operatorname{span}\left\{f_{n}\right\}_{n=1}^{N}$. Denote the optimal lower bound for that frame by $A_{N}$ and the frame operator by

$$
S_{N}: \operatorname{span}\left\{f_{n}\right\}_{n=1}^{N} \rightarrow \operatorname{span}\left\{f_{n}\right\}_{n=1}^{N}, S_{N} f=\sum_{n=1}^{N}<f, f_{n}>f_{n} .
$$

The equivalence between (i), (ii) and (iii) below is due to Kun and Lim [41] (a fast direct proof of (ii) $\Rightarrow$ (i) is in [11, and (iii) $\Rightarrow$ (i) is in 22]) and the equivalence between (iii) and (iv) is a consequence of the sequence $\left\{A_{N}\right\}_{N=1}^{\infty}$ being decreasing when $\left\{f_{n}\right\}_{n=1}^{\infty}$ is linearly independent. When we speak about a family $\left\{f_{n}\right\}_{n=1}^{\infty}$ being linearly independent, it means that every finite subfamily is linearly independent.

Theorem 2.9. Let $\left\{f_{n}\right\}_{n=1}^{\infty}$ be a frame for $\mathcal{H}$. For $N \in \mathbb{N}$, let $A_{N}$ denote the optimal lower frame bound for $\left\{f_{n}\right\}_{n=1}^{N}$. Then the following are equivalent:

(i) $\left\{f_{n}\right\}_{n=1}^{\infty}$ is a Riesz basis for $\mathcal{H}$.

(ii) $\left\{f_{n}\right\}_{n=1}^{\infty}$ is linearly independent and $<f, S_{N}^{-1} f_{n}>\rightarrow<f, S^{-1} f_{n}>$ as $N \rightarrow$ $\infty, \forall f \in \mathcal{H}, \forall n \in \mathbb{N}$.

(iii) $\left\{f_{n}\right\}_{n=1}^{\infty}$ is linearly independent and $\inf _{N \in \mathbb{N}} A_{N}>0$.

(iv) $\left\{f_{n}\right\}_{n=1}^{\infty}$ is linearly independent and $\lim _{N \rightarrow \infty} A_{N}$ exists and is positive.

Remark. An important consequence of Theorem 2.9 is stated in Theorem 4.7 The condition (ii) in Theorem 2.9] is motivated by the observation that it usually is difficult to invert the frame operator $S$, which makes it hard to find the frame coefficients $<f, S^{-1} f_{n}>$. Thus it is natural to try to approximate the frame coefficients using coefficients that are "easy" to calculate. The coefficients $<f, S_{N}^{-1} f_{n}>$ are "easy" to calculate in the sense that $S_{N}^{-1}$ can be found using finite-dimensional linear algebra, but in light of Theorem $2.9<f, S_{N}^{-1} f_{n}>$ is not well suited to approximate $<f, S^{-1} f_{n}>$ for general frames (most of the frames of interest turn out to be linearly independent; cf. Section 4.3). However, the approximation works for Riesz frames. In [16] a "regularized version" is presented, which works for all frames.

\section{Frames OF EXPONENTIALS}

Recall that the functions $\left\{\frac{1}{\sqrt{2 \pi}} e^{i m x}\right\}_{m \in \mathbb{Z}}$ constitute an orthonormal basis for $L^{2}(-\pi, \pi)$. Thus $\left\{e^{i m x}\right\}_{m \in \mathbb{Z}}$ is a frame for $L^{2}(-\pi, \pi)$ with bounds $A=B=2 \pi$. More generally, for $\left\{\lambda_{m}\right\}_{m \in \mathbb{Z}} \subseteq \mathbb{R}$, a frame for $L^{2}(-\pi, \pi)$ of the form $\left\{e^{i \lambda_{m} x}\right\}_{m \in \mathbb{Z}}$ is called a frame of exponentials and an expansion

$$
f(x) \sim \sum c_{m} e^{i m x}
$$

is called a nonharmonic Fourier series. Recall that this is the context in which frames were originally defined.

In this section we present some of the recent results for frames of exponentials. We mainly include this section as background for the study of Gabor frames, so we do not go into much detail.

3.1. Necessary and sufficient conditions. A sequence $\left\{\lambda_{m}\right\}_{m \in \mathbb{Z}} \subseteq \mathbb{R}$ is said to be $\delta$-separated if for all $m \neq n$,

$$
\left|\lambda_{m}-\lambda_{n}\right| \geq \delta>0
$$


If $\left\{\lambda_{m}\right\}_{m \in \mathbb{Z}}$ is a finite union of separated sets, we say that $\left\{\lambda_{m}\right\}_{m \in \mathbb{Z}}$ is relatively separated. A relatively separated sequence can repeat the same point a finite number of times, but it cannot have an accumulating point. It is not hard to prove directly that $\left\{e^{i \lambda_{m} x}\right\}_{m \in \mathbb{Z}}$ satisfies the upper frame condition if and only if $\left\{\lambda_{m}\right\}_{m \in \mathbb{Z}}$ is relatively separated.

In order for $\left\{e^{i \lambda_{m} x}\right\}_{m \in \mathbb{Z}}$ to be a frame for $L^{2}(-\pi, \pi),\left\{\lambda_{m}\right\}_{m \in \mathbb{Z}}$ has to satisfy certain density conditions. Given a number $r>0$, let $n^{-}(r)$ denote the minimal number of points from $\left\{\lambda_{m}\right\}_{m \in \mathbb{Z}}$ to be found in an interval of length $r$. The lower Beurling density of $\left\{\lambda_{m}\right\}_{m \in \mathbb{Z}}$ is defined by

$$
D^{-}\left(\left\{\lambda_{m}\right\}_{m \in \mathbb{Z}}\right):=\lim _{r \rightarrow \infty} \frac{n^{-}(r)}{r} .
$$

A fundamental result by Jaffard [39] and Seip 47] "almost" characterizes the frame properties of $\left\{e^{i \lambda_{m} x}\right\}_{m \in \mathbb{Z}}$ in terms of $D^{-}\left(\left\{\lambda_{m}\right\}_{m \in \mathbb{Z}}\right)$ :

Theorem 3.1. For $\left\{e^{i \lambda_{m} x}\right\}_{m \in \mathbb{Z}}$ to be a frame, it is necessary that $\left\{\lambda_{m}\right\}_{m \in \mathbb{Z}}$ is relatively separated and $D^{-}\left(\left\{\lambda_{m}\right\}_{m \in \mathbb{Z}}\right) \geq 1$, and it is sufficient that $\left\{\lambda_{m}\right\}_{m \in \mathbb{Z}}$ is relatively separated and $D^{-}\left(\left\{\lambda_{m}\right\}_{m \in \mathbb{Z}}\right)>1$.

Seip also proves that if $\left\{\lambda_{m}\right\}_{m \in \mathbb{Z}}$ is separated and $D^{-}\left(\left\{\lambda_{m}\right\}_{m \in \mathbb{Z}}\right)>1$, then $\left\{e^{i \lambda_{m} x}\right\}_{m \in \mathbb{Z}}$ contains a Riesz basis.

Theorem 3.1 is optimal in the sense that no conclusion is possible if $D^{-}\left(\left\{\lambda_{m}\right\}_{m \in \mathbb{Z}}\right)$ $=1$. For example, Seip proves that the sequence $\left\{\lambda_{m}\right\}=\left\{m\left(1-|m|^{-1 / 2}\right)\right\}_{|m|>1}$ has density 1 and that $\left\{e^{i \lambda_{m} x}\right\}_{m \in \mathbb{Z}}$ is a frame for $L^{2}(-\pi, \pi)$. On the other hand, the famous example of Kadec (cf. the discussion below),

$$
\lambda_{m}:= \begin{cases}m-1 / 4 & \text { if } m>0 \\ m+1 / 4 & \text { if } m<0 \\ 0 & \text { if } m=0\end{cases}
$$

also gives a sequence with density 1, however, without generating a frame for $L^{2}(-\pi, \pi)$. For a discussion of this example we refer to [51].

It is interesting to observe that for a family $\left\{\lambda_{m}\right\}_{m \in \mathbb{Z}}$, for which $\lambda_{m} \neq \lambda_{n}$ whenever $m \neq n$, the existence of a lower Riesz bound for $\left\{e^{i \lambda_{m} x}\right\}_{m \in \mathbb{Z}}$, i.e., a number $A>0$ such that

$$
A \sum\left|c_{m}\right|^{2} \leq \| \sum c_{m} e^{i \lambda_{m} \cdot \|^{2}}
$$

for all finite sequences $\left\{c_{m}\right\}$, automatically implies that $\left\{e^{i \lambda_{m} x}\right\}_{m \in \mathbb{Z}}$ is a Bessel sequence. That is, the condition (9) is enough to guarantee that $\left\{e^{i \lambda_{m} x}\right\}_{m \in \mathbb{Z}}$ is a Riesz basis for its closed span! This is a recent result by Lindner [43. Since Lindner's proof is very short and elegant, we include it below.

Theorem 3.2. Suppose that $\left\{\lambda_{m}\right\}_{m \in \mathbb{Z}} \subseteq \mathbb{R}$ consists of distinct points and that there exists a constant $A>0$ such that (9) is satisfied. Then $\left\{e^{i \lambda_{m} x}\right\}_{m \in \mathbb{Z}}$ is a Riesz basis for its closed span.

Proof. Consider $\lambda_{m}, \lambda_{n}$, where $n \neq m$. By assumption,

$$
2 A=A\left(|1|^{2}+|-1|^{2}\right) \leq\left\|e^{i \lambda_{m} x}-e^{i \lambda_{n} x}\right\|^{2}=\int_{-\pi}^{\pi}\left|1-e^{i\left(\lambda_{m}-\lambda_{n}\right) x}\right|^{2} d x .
$$


Now, for $x \in]-\pi, \pi]$,

$$
\left|1-e^{i\left(\lambda_{m}-\lambda_{n}\right) x}\right|=\left|-\sum_{k=1}^{\infty} \frac{\left[i\left(\lambda_{m}-\lambda_{n}\right) x\right]^{k}}{k !}\right| \leq e^{\left.\mid \lambda_{m}-\lambda_{n}\right) \mid \cdot \pi}-1 .
$$

It follows that $2 A \leq 2 \pi\left(e^{\left|\lambda_{m}-\lambda_{n}\right| \cdot \pi}-1\right)^{2}$, implying that

$$
\left|\lambda_{m}-\lambda_{n}\right| \geq \frac{1}{\pi} \ln \left(\sqrt{\frac{A}{\pi}}+1\right) .
$$

Thus $\left\{\lambda_{m}\right\}_{m \in \mathbb{Z}}$ is separated, and therefore $\left\{e^{i \lambda_{m} x}\right\}_{m \in \mathbb{Z}}$ is a Bessel sequence.

3.2. Kadec's 1/4-Theorem. The famous Kadec 1/4-Theorem [51] states that if $\left\{\lambda_{m}\right\}_{m \in \mathbb{Z}} \subseteq \mathbb{R}$ and $\sup _{m \in \mathbb{Z}}\left|\lambda_{m}-m\right|<\frac{1}{4}$, then $\left\{e^{i \lambda_{m} x}\right\}_{m \in \mathbb{Z}}$ is a Riesz basis for $L^{2}(-\pi, \pi)$. The example of Kadec (see (8)) shows that the conclusion fails if $\sup \left|\lambda_{m}-m\right|=\frac{1}{4}$. Combining the proof of Kadec's Theorem in [51] with the perturbation results for frames in [19], it is an easy matter to extend the result to frames; cf. [1], [15].

Theorem 3.3. Let $\left\{\lambda_{m}\right\}_{m \in \mathbb{Z}},\left\{\mu_{m}\right\}_{m \in \mathbb{Z}} \subseteq \mathbb{R}$. Suppose that $\left\{e^{i \mu_{m} x}\right\}_{m \in \mathbb{Z}}$ is a frame for $L^{2}(-\pi, \pi)$ with bounds $A, B$. If there exists a constant $L<1 / 4$ such that

$$
\left|\mu_{m}-\lambda_{m}\right| \leq L \forall m, \text { and } 1-\cos \pi L+\sin \pi L<\sqrt{\frac{A}{B}},
$$

then $\left\{e^{i \lambda_{m} x}\right\}_{m \in \mathbb{Z}}$ is a frame for $L^{2}(-\pi, \pi)$ with bounds

$$
A\left(1-\sqrt{\frac{B}{A}}(1-\cos \pi L+\sin \pi L)\right)^{2}, \quad B(2-\cos \pi L+\sin \pi L)^{2} .
$$

The advantage of this theorem compared to the original version also lies in the fact that we obtain frame bounds. Good values for the frame bounds are essential for estimates of the speed of convergence in algorithms involving frames; cf. 33. Recently, Theorem 3.3 has been used to construct Riesz bases for weighted $L^{2}$ spaces consisting of solutions to certain Sturm-Liouville problems; cf. [35].

\section{GABOR FRAmeS}

As mentioned in the last section, the fact that $\left\{\frac{1}{\sqrt{2 \pi}} e^{i m x}\right\}_{m \in \mathbb{Z}}$ constitutes an ONB for $L^{2}(-\pi, \pi)$ is the starting point for the theory for frames of exponentials. By a change of variable, $\left\{e^{i 2 \pi m x}\right\}_{m \in \mathbb{Z}}$ constitutes an ONB for $L^{2}(0,1)$. Observe that we can easily obtain an orthonormal basis for $L^{2}(\mathbb{R})$ by cutting $\mathbb{R}$ into intervals of length 1 and taking an orthonormal basis corresponding to each interval. More precisely, the set of functions

$$
\left\{e^{i 2 \pi m(x-n)} \mathbf{1}_{0,1[}(x-n)\right\}_{m, n \in \mathbb{Z}}=\left\{e^{i 2 \pi m x} \mathbf{1}_{0,1[}(x-n)\right\}_{m, n \in \mathbb{Z}}
$$

is an orthonormal basis for $L^{2}(\mathbb{R})$. This leads to the general definition of a Gabor frame: given parameters $a, b \in \mathbb{R}$ and a function $g \in L^{2}(\mathbb{R})$, a frame for $L^{2}(\mathbb{R})$ of the form $\left\{e^{i 2 \pi m b x} g(x-n a)\right\}_{m, n \in \mathbb{Z}}$ is called a Gabor frame. The term "Weyl-Heisenberg frame" is also used. Using the operators "translation" resp. "modulation" acting on functions $g \in L^{2}(\mathbb{R})$ by

$$
\left(T_{a} g\right)(x)=g(x-a), a>0, \operatorname{resp} .\left(E_{b} g\right)(x)=e^{2 \pi i b x} g(x), b>0,
$$

a Gabor frame can be written

$$
\left\{e^{i 2 \pi m b x} g(x-n a)\right\}_{m, n \in \mathbb{Z}}=\left\{E_{m b} T_{n a} g\right\}_{m, n \in \mathbb{Z}} .
$$


The origin of Gabor frames goes back to the paper 32, where Gabor proposes expanding signals $f$ as a series $f(x)=\sum c_{m, n} e^{i 2 \pi m b x} g(x-n a)$, where $g$ is the Gaussian. The idea was to use the expansion for communications: instead of transmitting the function $f$, one could send the coefficients $c_{m, n}$. The original idea has apparently not been developed very far, but Gabor frames have proved very useful in many other contexts. For a collection of research articles about Gabor systems (theory and applications) we refer to [31]. Another important source of information is the book 34 by Gröchenig, where Gabor frames are used in the context of time-frequency analysis.

4.1. Sufficient conditions. Sufficient conditions for $\left\{E_{m b} T_{n a} g\right\}_{m, n \in \mathbb{Z}}$ to be a frame for $L^{2}(\mathbb{R})$ have been known for about 10 years; cf. 37], Theorem 4.1.5:

Theorem 4.1. Let $g \in L^{2}(\mathbb{R})$ and suppose that there exist constants $A, B>0$ such that

(10) $\sum_{k \neq 0}\left\|\sum_{n \in \mathbb{Z}} T_{n a} g T_{n a+\frac{k}{b}} \bar{g}\right\|_{\infty}<A \leq \sum_{n \in \mathbb{Z}}|g(x-n a)|^{2} \leq B$ for a.e. $x \in \mathbb{R}$.

Then $\left\{E_{m b} T_{n a} g\right\}_{m, n \in \mathbb{Z}}$ is a frame for $L^{2}(\mathbb{R})$.

In particular, the condition (10) implies that

$$
\sup _{x} \sum_{k \neq 0}\left|\sum_{n \in \mathbb{Z}} g(x-n a) \overline{g(x-n a-k / b)}\right|<\inf _{x} \sum_{n \in \mathbb{Z}}|g(x-n a)|^{2} .
$$

Later, Ron and Shen [46] were able to give a complete characterization of Gabor frames. Given $g \in L^{2}(\mathbb{R})$, consider the matrix-valued function

$$
M(t):=(g(t-n a-m / b))_{m, n \in \mathbb{Z}} .
$$

Observe that $M(t) M(t)^{*}$ is a positive matrix.

Theorem 4.2. $\left\{E_{m b} T_{n a} g\right\}_{m, n \in \mathbb{Z}}$ is a frame for $L^{2}(\mathbb{R})$ with bounds $A, B$ if and only if

$$
A I \leq M(t) M(t)^{*} \leq B I \text { a.e. }
$$

Theorem 4.2 is related to another interesting result by Ron and Shen, saying that $\left\{E_{m b} T_{n a} g\right\}_{m, n \in \mathbb{Z}}$ is a frame for $L^{2}(\mathbb{R})$ if and only if $\left\{E_{\frac{m}{a}} T_{\frac{n}{b}} g\right\}_{m, n \in \mathbb{Z}}$ is a Riesz basis for its closed span. We refer to [46].

Theorem 4.2 is difficult to apply in its full generality. However, the condition on the matrix $M(t) M(t)^{*}$ is in particular satisfied if it is diagonal dominant. This leads to a sufficient condition for $\left\{E_{m b} T_{n a} g\right\}_{m, n \in \mathbb{Z}}$ to be a frame; see Theorem 4.3 below. An independent proof (which even delivers Gabor frames for subspaces of $L^{2}(\mathbb{R})$ ) can be found in [8]. The advantage of the result compared to Theorem 4.1 is that (10) is replaced by a condition comparing $\sum_{k \neq 0}\left|\sum_{n \in \mathbb{Z}} g(x-n a) \overline{g(x-n a-k / b)}\right|$ and $\sum_{n \in \mathbb{Z}}|g(x-n a)|^{2}$ pointwise. The formulation below is taken from [8].

Theorem 4.3. Let $g \in L^{2}(R), a, b>0$ and suppose that

$$
\begin{aligned}
& A:=\inf _{x \in[0, a]}\left[\sum_{n \in \mathbb{Z}}|g(x-n a)|^{2}-\sum_{k \neq 0}\left|\sum_{n \in \mathbb{Z}} g(x-n a) \overline{g\left(x-n a-\frac{k}{b}\right)}\right|\right]>0 \\
& B:=\sup _{x \in[0, a]} \sum_{k \in \mathbb{Z}}\left|\sum_{n \in \mathbb{Z}} g(x-n a) \overline{g\left(x-n a-\frac{k}{b}\right)}\right|<\infty \text {. }
\end{aligned}
$$


Then $\left\{E_{m b} T_{n a} g\right\}_{m, n \in \mathbb{Z}}$ is a frame for $L^{2}(\mathbb{R})$ with bounds $\frac{A}{b}, \frac{B}{b}$.

It is easy to find examples where Theorem 4.3 can be applied but where the condition in Theorem 4.1] is not satisfied; cf. [8]:

Example 4.4. Let $a=b=1$ and define

$$
g(x)= \begin{cases}1+x & \text { if } x \in[0,1[ \\ \frac{1}{2} x & \text { if } x \in[1,2[ \\ 0 & \text { otherwise. }\end{cases}
$$

For $x \in[0,1[$ we have

$$
\sum_{n \in \mathbb{Z}}|g(x-n)|^{2}=g(x)^{2}+g(x+1)^{2}=\frac{5}{4}(x+1)^{2}
$$

and

$$
\sum_{k \neq 0}\left|\sum_{n \in \mathbb{Z}} g(x-n) \overline{g(x-n-k)}\right|=(1+x)^{2},
$$

so by Theorem $4.3\left\{E_{m} T_{n} g\right\}_{m, n \in \mathbb{Z}}$ is a frame for $L^{2}(\mathbb{R})$ with bounds $A=\frac{1}{4}, B=9$. But $\inf _{x \in[0,1]} \sum_{n \in \mathbb{Z}}|g(x-n)|^{2}=\frac{5}{4}$ and

$$
\sum_{k \neq 0}\left\|\sum_{n \in \mathbb{Z}} T_{n} g T_{n+k} \bar{g}\right\|_{\infty}=4
$$

so the condition (10) is not satisfied.

It is interesting to observe that the frame properties for $\left\{E_{m b} T_{n a} g\right\}_{m, n \in \mathbb{Z}}$ depend heavily on the numbers $a, b$. Feichtinger and Janssen [30] have constructed a function $g \in L^{2}(\mathbb{R})$ for which $\left\{E_{m b} T_{n a} g\right\}_{m, n \in \mathbb{Z}}$ satisfies the upper frame condition for any two rationals $a, b>0$, while for any $\beta>0$ and any rational $c>0$ the family $\left\{E_{m \beta} T_{n c \alpha} g\right\}_{m, n \in \mathbb{Z}}$ does not satisfy the upper frame condition.

4.2. The Balian-Low Theorem and Wilson bases. We define the Fourier transform of $g \in L^{1}(\mathbb{R})$ by

$$
\hat{g}(\gamma)=\int_{\mathbb{R}} g(x) e^{-2 \pi i \gamma x} d x
$$

As usual, the Fourier transform is extended to a unitary mapping of $L^{2}(\mathbb{R})$ onto $L^{2}(\mathbb{R})$.

A famous result about Gabor frames states that $\left\{E_{m b} T_{n a} g\right\}_{m, n \in \mathbb{Z}}$ can only be a frame for $L^{2}(\mathbb{R})$ if $a b \leq 1$; and if $\left\{E_{m b} T_{n a} g\right\}_{m, n \in \mathbb{Z}}$ is a frame, it is a Riesz basis if and only if $a b=1$ (see [37], p. 657). For a Riesz basis $\left\{E_{m b} T_{n a} g\right\}_{m, n \in \mathbb{Z}}$, the Balian-Low Theorem ([37, Th. 4.3.7) states that

$$
\left(\int_{\mathbb{R}}|x g(x)|^{2} d x\right)\left(\int_{\mathbb{R}}|\gamma \hat{g}(\gamma)|^{2} d \gamma\right)=\infty
$$

In words, the Balian-Low Theorem means that a function $g$ generating a Gabor Riesz basis cannot be well localized in both time and frequency. In particular, the Gaussian $g(x)=e^{-x^{2}}$ does not generate a Gabor Riesz basis for $a b=1$. On the other hand, it has been proved by Seip and Wallsten that the Gaussian generates a frame whenever $a b<1$; cf. [48, [49]. The fact that one can construct Gabor frames that are well localized in both time and frequency is just one motivation for the study of those frames. 
Daubechies, Jaffard and Journé [27] have proved that if one is ready to give up the Gabor structure, it is possible to obtain a well localized orthonormal basis: if $g \in L^{2}(\mathbb{R})$ is chosen such that $\hat{g}$ is real valued and $\left\{E_{m} T_{n / 2} g\right\}_{m, n \in \mathbb{Z}}$ is a frame with bounds $A=B=2$, then the collection of functions $\left\{\psi_{l, k}\right\}_{l \geq 0, k \in \mathbb{Z}}$ defined by

$$
\psi_{l, k}(x)= \begin{cases}g(x-k) & \text { for } \quad l=0 \\ \sqrt{2} g(x-k / 2) \cos (2 \pi l x) & \text { for } l>0, k+l \text { even } \\ \sqrt{2} g(x-k / 2) \sin (2 \pi l x) & \text { for } \quad l>0, k+l \text { odd }\end{cases}
$$

constitutes an orthonormal basis for $L^{2}(\mathbb{R}) !\left\{\psi_{l, k}\right\}_{l \geq 0, k \in \mathbb{Z}}$ is called a Wilson basis. Observe that

$$
\psi_{l, k}= \begin{cases}E_{0} T_{k} g & \text { for } \quad l=0 \\ \frac{1}{\sqrt{2}}\left(E_{l} T_{k / 2} g+E_{-l} T_{k / 2} g\right) & \text { for } \quad l>0, k+l \text { even } \\ \frac{-i}{\sqrt{2}}\left(E_{l} T_{k / 2} g-E_{-l} T_{k / 2} g\right) & \text { for } \quad l>0, k+l \text { odd; }\end{cases}
$$

i.e., the functions in the Wilson basis consist of linear combinations of the functions in the Gabor system $\left\{E_{m} T_{n / 2} g\right\}_{m, n \in \mathbb{Z}}$. By choosing $g$ such that

$$
\left(\int_{\mathbb{R}}|x g(x)|^{2} d x\right)\left(\int_{\mathbb{R}}|\gamma \hat{g}(\gamma)|^{2} d \gamma\right)<\infty,
$$

we have obtained an orthonormal basis circumventing the Balian-Low Theorem. We refer to [3] and 27] for more information, especially to [27] for a construction of a suitable function $g$.

Observe that the important feature of the system $\left\{\psi_{l, k}\right\}_{l \geq 0, k \in \mathbb{Z}}$ is that it is an orthonormal basis. It is not complicated to construct frames with a similar structure:

Proposition 4.5. Suppose that $\left\{E_{m b} T_{n a} g\right\}_{m, n \in \mathbb{Z}}$ is a frame with upper bound $B$. Then the collection of functions

$\left\{g_{n}\right\}_{n \in I}=\{g(x-n a)\}_{n \in \mathbb{Z}} \cup\{\cos (2 \pi m b x) g(x-n a), \sin (2 \pi m b x) g(x-n a)\}_{m \in \mathbb{N}, n \in \mathbb{Z}}$ is a frame for $L^{2}(\mathbb{R})$ with upper bound $B$.

For the proof, it is easy to see that the pre-frame operator corresponding to $\left\{g_{n}\right\}_{n \in I}$ in Proposition 4.5 is bounded and surjective, so the result follows by Theorem 2.5 .

4.3. Irregular Gabor systems and open problems. In connection with a Gabor system $\left\{E_{m b} T_{n a} g\right\}_{m, n \in \mathbb{Z}}$, one usually thinks about the points $(m b, n a)$ as a lattice in $\mathbb{R}^{2}$. Given a sequence of distinct points $\left\{\left(\lambda_{n}, \mu_{n}\right)\right\} \subseteq \mathbb{R}^{2}$, one could also consider the family $\left\{e^{i 2 \pi \lambda_{n} x} g\left(x-\mu_{n}\right)\right\}$, which is usually called an irregular Gabor system. Very little is known about frame properties of $\left\{e^{i 2 \pi \lambda_{n} x} g\left(x-\mu_{n}\right)\right\}$. The purpose of this section is to present some of the known results and point out some of the open problems.

By letting $|\cdot|$ denote the euclidian norm in $\mathbb{R}^{2}$, the definition of a relatively separated set from Section 3 can also be used for a set $\left\{\lambda_{m}\right\}_{m \in \mathbb{Z}} \subseteq \mathbb{R}^{2}$. By letting $n^{-}(r)$ denote the minimal number of points from $\left\{\lambda_{m}\right\}_{m \in \mathbb{Z}}$ to be found in a ball with radius $r / 2$, the lower density is defined by

$$
D^{-}\left(\left\{\lambda_{m}\right\}_{m \in \mathbb{Z}}\right)=\lim _{r \rightarrow \infty} \frac{n^{-}(r)}{r^{2}} .
$$


Christensen, Deng and Heil proved that for $\left\{e^{i 2 \pi \lambda_{n} x} g\left(x-\mu_{n}\right)\right\}$ to be a frame, it is necessary that $\left\{\left(\lambda_{n}, \mu_{n}\right)\right\}$ is relatively separated and that $D^{-}\left(\left\{\left(\lambda_{n}, \mu_{n}\right)\right\}\right) \geq 1$; cf. [20], Theorems 1.1, 3.1. For a regular Gabor system $\left\{E_{m b} T_{n a} g\right\}_{m, n \in \mathbb{Z}}$ the latter assumption corresponds exactly to the condition $a b \leq 1$. It is known that if $a b>1$, then $\left\{E_{m b} T_{n a} g\right\}_{m, n \in \mathbb{Z}}$ is not complete in $L^{2}(\mathbb{R})$. One could therefore expect that $\left\{e^{i 2 \pi \lambda_{n} x} g\left(x-\mu_{n}\right)\right\}$ must be incomplete whenever $D^{-}\left(\left\{\left(\lambda_{n}, \mu_{n}\right)\right\}\right)<1$. However, Benedetto, Heil and Walnut have shown that this is false, by construction of sets $\left\{\left(\lambda_{n}, \mu_{n}\right)\right\}$ with arbitrarily small density and yet such that $\left\{e^{i 2 \pi \lambda_{n} x} g\left(x-\mu_{n}\right)\right\}$ is complete; cf. [3], Th. 2.6.

Also, Ramanathan and Steger have proved that the density must be exactly one in order to obtain a Riesz basis; cf. [45], Cor. 4.

No practically useful condition for $\left\{e^{i 2 \pi \lambda_{n} x} g\left(x-\mu_{n}\right)\right\}$ to be a frame is known. But based on an abstract theory for atomic decomposition, Feichtinger and Gröchenig [29] have shown that for $g$ "sufficiently nice", every "well-spread" family $\left\{\left(\lambda_{n}, \mu_{n}\right)\right\}$ will give rise to a Gabor frame:

Theorem 4.6. Choose a non-zero function $g \in L^{2}(\mathbb{R})$ such that

$$
\int_{\mathbb{R}} \int_{\mathbb{R}}\left|<E_{x} M_{y} g, g>\right| d x d y<\infty .
$$

Then there exists an open set $U \subset \mathbb{R}^{2}$ such that $\left\{e^{i 2 \pi \lambda_{n} x} g\left(x-\mu_{n}\right)\right\}$ is a frame for $L^{2}(\mathbb{R})$ for every separated set $\left\{\left(\lambda_{n}, \mu_{n}\right)\right\} \subseteq \mathbb{R}^{2}$ for which

$$
\bigcup_{n}\left[\left(\lambda_{n}, \mu_{n}\right)+U\right]=\mathbb{R}^{2} .
$$

We refer to 17] for a unified presentation of the Feichtinger/Gröchenig theory and the proof of Theorem 4.6. It can be proved that (13) is satisfied for a dense set of functions $g \in L^{2}(\mathbb{R})$. Unfortunately the Feichtinger/Gröchenig theory does not give much information about how to choose an appropriate set $U$, so Theorem 4.6 is at the moment mainly theoretically interesting. However, the Feichtinger/Gröchenig theory has many attractive features (it actually delivers discrete expansions in a very large class of Banach spaces), and it would certainly be worthwhile to search for easily verifiable conditions for the theory to apply.

Let us end this section with a few words about a conjecture by Heil, Ramanathan and Topiwala [36]: it says that if $\left\{\left(\lambda_{n}, \mu_{n}\right)\right\}$ is a finite set of distinct points and $g$ is a non-zero function in $L^{2}(\mathbb{R})$, then $\left\{e^{i 2 \pi \lambda_{n} x} g\left(x-\mu_{n}\right)\right\}$ is linearly independent. The conjecture is proved under some extra assumptions in 36. Later, Linnell 44 was able to prove it in the case where $\left\{\left(\lambda_{n}, \mu_{n}\right)\right\}$ is a lattice (or a subset thereof), i.e., for

$$
\left\{\left(\lambda_{n}, \mu_{n}\right)\right\}=\{(m b, n a)\}_{n=1, m=1}^{N, M} .
$$

Thus, finite subsets of a Gabor frame $\left\{E_{m b} T_{n a} g\right\}_{m, n \in \mathbb{Z}}$ for $L^{2}(\mathbb{R})$ will be linearly independent. The general conjecture is still open.

Intuitively, one would expect the optimal lower frame bound for a finite subfamily $\left\{E_{m b} T_{n a} g\right\}_{m, n=1}^{N}$ of a Gabor frame $\left\{E_{m b} T_{n a} g\right\}_{m, n \in \mathbb{Z}}$ to converge to the optimal lower bound for $\left\{E_{m b} T_{n a} g\right\}_{m, n \in \mathbb{Z}}$ when the subfamily gets larger, i.e., for $N \rightarrow \infty$. Surprisingly, this is not true: 
Theorem 4.7. Suppose that $a b<1$ and that $\left\{E_{m b} T_{n a} g\right\}_{m, n \in \mathbb{Z}}$ is a frame for $L^{2}(\mathbb{R})$. Let $A_{N}$ denote the optimal lower bound for $\left\{E_{m b} T_{n a} g\right\}_{m, n=1}^{N}$. Then

$$
A_{N} \rightarrow 0 \text { as } N \rightarrow \infty \text {. }
$$

Proof. As observed before, the assumption $a b<1$ implies that $\left\{E_{m b} T_{n a} g\right\}_{m, n \in \mathbb{Z}}$ is not a Riesz basis. By Linnell's result, $\left\{E_{m b} T_{n a} g\right\}_{m, n \in \mathbb{Z}}$ is linearly independent. By Theorem 2.9] we conclude that $A_{N} \rightarrow 0$ as $N \rightarrow \infty$.

\section{WAVELET FRAMES}

The dilation operators $D_{a}$ acting on $\psi \in L^{2}(\mathbb{R})$ are defined by

$$
\left(D_{a} \psi\right)(x)=\frac{1}{\sqrt{a}} \psi\left(\frac{x}{a}\right), x \in \mathbb{R}, a>0 .
$$

From the mathematical point of view, the main question in wavelet analysis is to construct a function $\psi$ such that

$$
\left\{D_{a^{j}} T_{b k} \psi(x)\right\}_{j, k \in \mathbb{Z}}=\left\{\frac{1}{a^{j / 2}} \psi\left(\frac{x}{a^{j}}-k b\right)\right\}_{j, k \in \mathbb{Z}}
$$

constitutes a frame (or Riesz basis) for $L^{2}(\mathbb{R})$ with prescribed properties. The importance of wavelets actually delivers a good argument for the study of frames. It is proved in [24] that no orthonormal basis $\left\{D_{a^{j}} T_{b k} \psi\right\}_{j, k \in \mathbb{Z}}$ having a $C^{\infty}$-mother wavelet $\psi$ which is exponentially decreasing exists. However, frames of this type exist! As an example, Daubechies mentions the famous Mexican hat wavelet

$$
\psi(x)=\left(1-x^{2}\right) e^{-x^{2} / 2} .
$$

It is known that the Mexican hat wavelet for a given value of $a>0$ generates a wavelet frame $\left\{D_{a^{j}} T_{b k} \psi\right\}_{j, k \in \mathbb{Z}}$ for all sufficiently small values of $b>0$.

The theory for wavelets has developed rapidly over the last 10 years, mainly due to the success of multiresolution analysis. There is a vast literature about multiresolution analysis, so we will not discuss that subject here but just refer to the classical monograph [25] by Daubechies and the more recent book [50] by Wojtaszczyk. Instead, we will discuss shortly the extension - the theory for frame multiresolution analysis - proposed by Benedetto and Li. For more information about wavelets in theory and practice we refer to the collections of research articles [2], 23].

5.1. A sufficient condition. A sufficient condition for $\left\{D_{a^{j}} T_{b k} \psi\right\}_{j, k \in \mathbb{Z}}$ to be a frame can be found in 37, Theorem 5.1.6. The generalization below is very similar to the way Theorem 4.1 was extended to Theorem4.3. It appeared (without proof) in $[8]$.

Theorem 5.1. Let $a>1, b>0$ and $\psi \in L^{2}(\mathbb{R})$ be given. Suppose that

$$
\begin{aligned}
& A:=\inf _{|\gamma| \in[1, a]}\left[\sum_{n \in \mathbb{Z}}\left|\hat{\psi}\left(a^{n} \gamma\right)\right|^{2}-\sum_{k \neq 0} \sum_{n \in \mathbb{Z}}\left|\hat{\psi}\left(a^{n} \gamma\right) \hat{\psi}\left(a^{n} \gamma+k / b\right)\right|\right]>0, \\
& B \quad:=\sup _{|\gamma| \in[1, a]} \sum_{k, n \in \mathbb{Z}}\left|\hat{\psi}\left(a^{n} \gamma\right) \hat{\psi}\left(a^{n} \gamma+k / b\right)\right|<\infty .
\end{aligned}
$$

Then $\left\{\frac{1}{a^{j / 2}} \psi\left(\frac{x}{a^{j}}-k b\right)\right\}_{j, k \in \mathbb{Z}}$ is a frame for $L^{2}(\mathbb{R})$ with bounds $\frac{A}{b}, \frac{B}{b}$. 
Proof. We only sketch the proof. The starting point is [37, Theorem 5.1.6, where it is proved that if $\hat{f}$ is a continuous function with compact support,

$$
\begin{aligned}
& \sum_{j, k \in \mathbb{Z}}\left|<f, D_{a^{j}} T_{k b} \psi>\right|^{2} \\
& =\frac{1}{b} \int_{\mathbb{R}}|\hat{f}(\gamma)|^{2} \sum_{n \in \mathbb{Z}}\left|\hat{\psi}\left(a^{n} \gamma\right)\right|^{2} d \gamma \\
& +\frac{1}{b} \sum_{k \neq 0} \sum_{n \in \mathbb{Z}} \int_{\mathbb{R}} \hat{f}(\gamma) \overline{\hat{f}\left(\gamma-a^{n} k / b\right) \hat{\psi}\left(a^{-n} \gamma\right)} \hat{\psi}\left(a^{-n} \gamma-k / b\right) d \gamma .
\end{aligned}
$$

Now, using Cauchy-Schwarz first on the integral and then on the sum over $k$, we obtain

$$
\begin{aligned}
R:= & \left|\sum_{k \neq 0} \sum_{n \in \mathbb{Z}} \int_{\mathbb{R}} \hat{f}(\gamma) \overline{\hat{f}\left(\gamma-a^{n} k / b\right) \hat{\psi}\left(a^{-n} \gamma\right)} \hat{\psi}\left(a^{-n} \gamma-k / b\right) d \gamma\right| \\
\leq & \sum_{n \in \mathbb{Z}}\left[\sum_{k \neq 0} \int_{\mathbb{R}}|\hat{f}(\gamma)|^{2} \cdot\left|\hat{\psi}\left(a^{-n} \gamma\right) \cdot \hat{\psi}\left(a^{-n} \gamma-k / b\right)\right| d \gamma\right]^{1 / 2} \\
& \times\left[\sum_{k \neq 0} \int_{\mathbb{R}}\left|\hat{f}\left(\gamma-a^{n} k / b\right)\right|^{2} \cdot\left|\hat{\psi}\left(a^{-n} \gamma\right)\right| \cdot \hat{\psi}\left(a^{-n} \gamma-k / b\right) \mid d \gamma\right]^{1 / 2} \\
= & \sum_{n \in \mathbb{Z}}(*)(* *) .
\end{aligned}
$$

The terms $\left({ }^{*}\right)$ and $\left({ }^{* *}\right)$ are actually identical (use the change of variable $\gamma \rightarrow$ $\gamma+a^{n} k / b$ in $\left.\left({ }^{* *}\right)\right)$, so by changing the summation index $n \rightarrow-n, k \rightarrow-k$,

$$
\begin{aligned}
R & \leq \sum_{n \in \mathbb{Z}} \sum_{k \neq 0} \int_{\mathbb{R}}|\hat{f}(\gamma)|^{2} \cdot\left|\hat{\psi}\left(a^{-n} \gamma\right) \cdot \hat{\psi}\left(a^{-n} \gamma-k / b\right)\right| d \gamma \\
& =\int_{\mathbb{R}}|\hat{f}(\gamma)|^{2} \sum_{k \neq 0} \sum_{n \in \mathbb{Z}}\left|\hat{\psi}\left(a^{n} \gamma\right) \hat{\psi}\left(a^{n} \gamma+k / b\right)\right| d \gamma
\end{aligned}
$$

Therefore, using the assumptions, we obtain

$$
\begin{aligned}
& \sum_{j, k \in \mathbb{Z}}\left|<f, D_{a^{j}} T_{k b} \psi>\right|^{2} \\
\geq & \frac{1}{b} \int_{\mathbb{R}}|\hat{f}(\gamma)|^{2}\left(\sum_{n \in \mathbb{Z}}\left|\hat{\psi}\left(a^{n} \gamma\right)\right|^{2}-\sum_{k \neq 0} \sum_{n \in \mathbb{Z}}\left|\hat{\psi}\left(a^{n} \gamma\right) \hat{\psi}\left(a^{n} \gamma+k / b\right)\right|\right) d \gamma \\
\geq & \frac{A}{b}\|f\|^{2}
\end{aligned}
$$

with a similar upper estimate. Since they hold on a dense subset of $L^{2}(\mathbb{R})$, the conclusion follows.

Remark. There is one remarkable difference between Theorem 5.1 and the similar result for Gabor frames (Theorem 4.3): in the condition for the lower bound in Theorem 4.3 it is the sum over $k$ of $\left|\sum_{n \in \mathbb{Z}} g(x-n a) \overline{g\left(x-n a-\frac{k}{b}\right)}\right|$ that has to be subtracted from $\sum_{n \in \mathbb{Z}}|g(x-n a)|^{2}$. That is, the absolute sign is outside the sum over $n$. This is in contrast to the condition in Theorem 5.1 where the absolute sign 
is inside the sum over $n$. The condition in Theorem 4.3 is clearly the best, since the position of the absolute sign opens up for possible cancellations. For $a=2$, it is known that the condition in Theorem 5.1 can be replaced with a condition where the absolute sign is outside; cf. [24], Theorem 2.9. It would be interesting if Theorem 5.1 above could be generalized that way.

5.2. Frame multiresolution analysis. Frame multiresolution analysis (FMRA) was introduced by Benedetto and $\mathrm{Li}$ [4. The purpose of the theory is to construct frames for $L^{2}(\mathbb{R})$ of the form $\left\{D_{2^{j}} T_{k} \psi\right\}_{j, k \in \mathbb{Z}}$. Formally, an FMRA is defined as a multiresolution analysis (MRA), with the condition " $\left\{T_{k} \phi\right\}_{k \in \mathbb{Z}}$ is a Riesz basis for $V_{0}$ " replaced by a frame condition:

Definition 5.2. An FMRA for $L^{2}(\mathbb{R})$ consists of a sequence of closed subspaces $\left\{V_{j}\right\}_{j \in \mathbb{Z}} \subseteq L^{2}(\mathbb{R})$ and a function $\phi \in V_{0}$ such that

(i) $\cdots V_{-1} \subseteq V_{0} \subseteq V_{1} \cdots$.

(ii) $\overline{\cup_{j} V_{j}}=L^{2}(\mathbb{R})$ and $\overline{\cap_{j} V_{j}}=0$.

(iii) $f \in V_{j} \Leftrightarrow[t \rightarrow f(2 t)] \in V_{j+1}$.

(iv) $f \in V_{0} \Rightarrow T_{k} f \in V_{0}, \forall k \in \mathbb{Z}$.

(v) $\left\{T_{k} \phi\right\}_{k \in \mathbb{Z}}$ is a frame for $V_{0}$.

The starting point for the construction of an FMRA is a function $\phi \in L^{2}(\mathbb{R})$ which is a frame for $\overline{\operatorname{span}}\left\{T_{k} \phi\right\}_{k \in \mathbb{Z}}$. Given $\phi \in L^{2}(\mathbb{R})$, define the function

$$
\Phi(x):=\sum_{k}|\hat{\phi}(x+k)|^{2} .
$$

Theorem 5.3. Let $\phi \in L^{2}(\mathbb{R})$. Then $\left\{T_{k} \phi\right\}_{k \in \mathbb{Z}}$ is a frame for $\overline{\operatorname{span}}\left\{T_{k} \phi\right\}_{k \in \mathbb{Z}}$ if and only if there exist constants $A, B>0$ such that

$$
A \leq \Phi(x) \leq B \text { a.e. on }\{x: \Phi(x) \neq 0\} .
$$

Theorem 5.3 is due to Benedetto and $\mathrm{Li}$ (with a superfluous assumption removed in 12]). In words, the condition means that $\sum_{k}|\hat{\phi}(x+k)|^{2}$ has to be bounded below and above, away from its zero-set. Given a function $\phi$ such that $\left\{T_{k} \phi\right\}_{k \in \mathbb{Z}}$ is a frame for $\overline{\operatorname{span}}\left\{T_{k} \phi\right\}_{k \in \mathbb{Z}}$, define the spaces $V_{j}$ by

$$
V_{0}=\overline{\operatorname{span}}\left\{T_{k} \phi\right\}_{k \in \mathbb{Z}}, \quad V_{j}=\left\{f \in L^{2}(\mathbb{R}) \mid\left[t \rightarrow f\left(2^{-j} t\right)\right] \in V_{0}\right\} .
$$

Observe that $V_{0}$ will always be a pure subspace of $L^{2}(\mathbb{R})$ : in [20] it is proved that translates of a single function never can constitute a frame for all of $L^{2}(\mathbb{R})$. A sufficient condition for $\left\{V_{j}, \phi\right\}$ to be an FMRA was given in [4, Theorem 4.6:

Theorem 5.4. Suppose that $\phi \in L^{2}(\mathbb{R})$, that $\left\{T_{k} \phi\right\}_{k \in \mathbb{Z}}$ is a frame for $V_{0}$, and that $|\hat{\phi}|>0$ in a neighborhood of zero. If there exists a function $H \in L^{\infty}(0,1)$ such that

$$
\hat{\phi}(\gamma)=\frac{1}{\sqrt{2}} H\left(\frac{\gamma}{2}\right) \hat{\phi}\left(\frac{\gamma}{2}\right)
$$

then $\left\{V_{j}, \phi\right\}$ is an FMRA.

Note the similarity between Theorem 5.4 and the results for an MRA; cf. [50], Th. 2.13 ! We now turn to the question about construction of a frame $\left\{D_{2^{j}} T_{k} \psi\right\}_{j, k \in \mathbb{Z}}$ based on a given FMRA. Let $W_{j}$ denote the orthogonal complement of $V_{j}$ in $V_{j+1}$. As for an MRA, the main question (when an FMRA has been constructed!) is to find a wavelet $\psi$ such that $\left\{T_{k} \psi\right\}_{k \in \mathbb{Z}}$ is a frame for $W_{0}$. This implies that $\left\{D_{2^{j}} T_{k} \psi\right\}_{j, k \in \mathbb{Z}}$ is a frame for $L^{2}(\mathbb{R})$; cf. [4], Theorem 5.11. 
Benedetto and Treiber [6] have proved that the existence of such a function $\psi$ depends solely on the "size" of a certain set $\Gamma$ :

Theorem 5.5. Let $\left\{V_{j}, \phi\right\}$ be an FMRA and let

$$
\Gamma:=\left\{x \in[0,1]: \Phi(2 x)=0, \Phi(x)>0, \Phi\left(x+\frac{1}{2}\right)>0\right\} .
$$

Then the following holds:

(i) If $\Gamma$ has positive Lebesgue measure, there does not exist a function $\psi \in L^{2}(\mathbb{R})$ such that $\left\{T_{k} \psi\right\}_{k \in \mathbb{Z}}$ is a frame for $W_{0}$.

(ii) If $\Gamma$ has vanishing Lebesgue measure, then there exists a function $\psi \in L^{2}(\mathbb{R})$ such that $\left\{T_{k} \psi\right\}_{k \in \mathbb{Z}}$ is a frame for $W_{0}$.

In the case (ii) Benedetto and Treiber also show how to define a suitable function $\psi$. Furthermore, given a candidate for $\psi$, one can always check whether $\left\{T_{k} \psi\right\}_{k \in \mathbb{Z}}$ is a frame using Theorem 5.3 .

For practical purposes the advantage of an FMRA compared to an MRA is that the underlying filter bank can be narrow band. We refer to [5] for a more detailed discussion.

Acknowledgment. The author would like to thank his coauthors during the last years, especially Pete Casazza, Christopher Heil and Alexander Lindner. The idea of writing this paper came from a Ph.D. course the author gave at San Luis University in Argentina in November 1999 (the course material 21] contains full proofs of several of the results in this survey). He is grateful to Sergio Favier and Felipe Zó for their help and support. He also thanks Hans Feichtinger, Christopher Heil, and Norbert Kaiblinger for many useful remarks.

\section{REFERENCES}

[1] Balan, R.: Stability theorems for Fourier frames and wavelet Riesz bases. J. Fourier Anal. and Appl. 3 (1997), p. 499-504. MR 99f:42060

[2] Benedetto J. and Frazier, M.: Wavelets: mathematics and applications. CRC Press, 1994. MR 94f: 42048

[3] Benedetto, J., Heil, C., and Walnut, D.: Differentiation and the Balian-Low theorem. J. Fourier Anal. Appl. 1 no.4 (1995), p. 355-402. MR 96f:42002

[4] Benedetto, J. and Li, S.: The theory of multiresolution analysis frames and applications to filter banks. Appl. Comp. Harm. Anal. 5 (1998), p. 389-427. MR 99k:42054

[5] Benedetto, J. and Li, S.: Subband coding and noise reduction in frame multiresolution analysis. In "Proceedings of SPIE Conference on Mathematical Imaging", San Diego, July 1994.

[6] Benedetto, J. and Treiber, O.: Wavelet frames: multiresolution analysis and extension principles. To appear in "Wavelet transforms and time-frequency signal analysis", ed. L. Debnath.

[7] Casazza, P.G. The art of frame theory. Taiwanese J. of Math. 4 no.2 (2000), p. 129-201. CMP 2000:13

[8] Casazza, P.G. and Christensen, O.: Weyl-Heisenberg frames for subspaces of $L^{2}(\mathbb{R})$, Proc. Amer. Math. Soc. 129 (2001), 145-154. CMP 2001:01

[9] Casazza, P.G. and Christensen, O.: Frames and Schauder bases. In "Approximation Theory: In Memory of A. K. Varna”, p. 133-139. Eds N. K. Govil, R. N. Mohapatra, Z. Nashed, A. Sharma, J. Szabados. Marcel Dekker, 1998. MR 99d:46030

[10] Casazza, P.G. and Christensen, O.: Frames containing a Riesz basis and preservation of this property under perturbation. SIAM J. Math. Anal. 29 no.1, 1998, p. 266-278. MR 99i:42043

[11] Casazza, P.G. and Christensen, O.: Approximation of the inverse frame operator and applications to Gabor frames. J. Approx. Theory 103 no.2 (2000), p. 338-356. MR 2001b:42037

[12] Casazza, P.G., Christensen, O., and Kalton, N.: Frames of translates. Collectanea Mathematica, to appear. 
[13] Christensen, O.: Frames and pseudo-inverses. J. Math. Anal. Appl. 195 (1995) , p. 401-414. MR 97c: 47003

[14] Christensen, O.: Frames and the projection method. Appl. Comp. Harm Anal., 1 (1993), p. 50-53. MR 95a:42041

[15] Christensen, O.: Operators with closed range, pseudo-inverses, and perturbation of frames for a subspace. Canad. Math. Bull 42 no.1 (1999) p. 37-45. MR 2000d:47003

[16] Christensen, O.: Finite-dimensional approximation of the inverse frame operator and applications to Weyl-Heisenberg frames and wavelet frames. J. Fourier Anal. Appl. 6 no. 1 (2000), p.79-91. CMP 2000:12

[17] Christensen, O.: Atomic decomposition via projective group representations. Rocky Mountain J. Math. 26 no. 4 (1996), p. 1289-1312. MR 98h:43004

[18] Christensen, O.: Frames containing a Riesz basis and approximation of the frame coefficients using finite dimensional methods. J. Math. Anal. Appl. 199 (1996), p. 256-270. MR 97b:46020

[19] Christensen, O.: A Paley-Wiener theorem for frames. Proc. Amer. Math. Soc. 123 (1995), p. 2199-2202. MR 95i:46027

[20] Christensen, O., Deng, B., and Heil, C.: Density of Gabor frames. Appl. Comp. Harm. Anal. 7 (1999), p. 292-304. MR 2000j:42043

[21] Christensen, O. and Jensen, T. K.: An introduction to the theory of bases, frames, and wavelets. Tech. Univ. of Denmark, 2000. 80 pages. Can be downloaded from the NuHAG homepage, http://tyche.mat.univie.ac.at.

[22] Christensen, O. and Lindner, A.: Frames of exponentials: lower frame bounds for finite subfamilies, and approximation of the inverse frame operator. Lin. Alg. and Its Appl. 323 (2001), p. 117-130.

[23] Chui, C.: Wavelets - a tutorial in theory and applications. Academic Press, 1992. MR 92k:42001

[24] Daubechies, I.: The wavelet transformation, time-frequency localization and signal analysis. IEEE Trans. Inform. Theory 36 (1990), p. 961- 1005. MR 91e:42038

[25] Daubechies, I.: Ten lectures on wavelets. CBMS-NSF Regional Conf. Ser. Appl. Math., 61. SIAM, Philadelphia, PA, 1992. MR 93e:42045

[26] Daubechies, I., Grossmann, A. and Meyer, Y.: Painless nonorthogonal expansions. J. Math. Phys. 27 (1986), p. 1271-1283. MR 87e:81089

[27] Daubechies, I. Jaffard, S. and Journé, J.L.: A simple Wilson orthonormal basis with exponential decay. SIAM J. Math. Anal. 22 (1991), p. 554-572. MR 92a:81066a

[28] Duffin, R.J. and Schaeffer, A.C.: A class of nonharmonic Fourier series. Trans. Amer. Math. Soc. 72 (1952) p. 341-366. MR 13:839a

[29] Feichtinger, H. G. and Gröchenig, K. H.: Banach spaces related to integrable group representations and their atomic decomposition. J. Funct. Anal. 86 (1989), p. 307-340. MR 91g:43011

[30] Feichtinger, H. G. and Janssen, A.J.E.M.: Validity of WH-frame bound conditions depends on lattice parameters. Appl. Comp. Harm. Anal. 8 no. 1 (2000), p. 104-112. MR 2000j:42044

[31] Feichtinger, H. G. and Strohmer, T., (Eds.): Gabor analysis and algorithms: Theory and applications. Birkhäuser Boston, 1998. MR 98h:42001

[32] Gabor, D.: Theory of communications. J. IEE (London) 93 no. 3 (1946), p. 429-457.

[33] Gröchenig, K. H.: Acceleration of the frame algorithm. IEEE Trans. Signal Processing 41 no. 12 (1993), p. 3331-3340.

[34] Gröchenig, K. H.: Foundations of time-frequency analysis. Birkhäuser, 2001.

[35] He, X. and Volkmer, H.: Riesz bases of solutions of Sturm-Liouville equations. J. Fourier Anal. Appl., to appear.

[36] Heil, C., Ramanathan, J. and Topiwala, P.: Linear independence of time-frequency translates. Proc. Amer. Math. Soc. 124 (1996), p. 2787-2795. MR 96k:42039

[37] Heil, C. and Walnut, D.: Continuous and discrete wavelet transforms. SIAM Review 31 (1989), p. 628-666. MR 91c:42032

[38] Heuser, H.: Functional Analysis. John Wiley, Chichester, 1982. MR 83m:46001

[39] Jaffard, S.: A density criterion for frames of complex exponentials. Michigan J. Math. 38 (1991), p. 339-348. MR 92i:42001

[40] Janssen, A.J.E.M.: Signal analytic proofs of two basic results on lattice expansions. Appl. Comp. Harm. Anal. 1 (1994), p. 350-354. MR 95m:42044

[41] Kim, H.O. and Lim, J.K.: New characterizations of Riesz bases. Appl. Comp. Harm. Anal. 4 (1997), p. 222-229. MR 98d:46020 
[42] Li, S.: On general frame decompositions. Numer. Funct. Anal. and Optimiz. 16 no. 9, 10 (1995), p. 1181-1191. MR 97b:42055

[43] Lindner, A.: A universal constant for exponential Riesz sequences. Z. Anal. Anwend. 19 (2000) no. 2, p. 553-559. CMP 2000:15

[44] Linnell, P.: Von Neumann algebras and linear independence of translates. Proc. Amer. Math. Soc. 127 no. 11 (1999), p. 3269-3277. MR 2000b:46106

[45] Ramanathan, J. and Steger, T.: Incompleteness of sparse coherent states. Appl. Comp. Harm. Anal. 2 (1995), p. 148-153. MR 96b:81049

[46] Ron, A. and Shen, Z.: Weyl-Heisenberg frames and Riesz bases in $L^{2}\left(\mathbb{R}^{d}\right)$. Duke Math. J. 89 (1997), p. 237-282. MR 98i:42013

[47] Seip, K.: On the connection between exponential bases and certain related sequences in $L^{2}(-\pi, \pi)$. J. Funct. Anal. 130 (1995), p. 131-160. MR 96d:46030

[48] Seip, K.: Density theorems for sampling and interpolation in the Bargmann-Fock space I. J. Reine Angew. Math. 429 (1992), p. 91-106. MR 93g:46026a

[49] Seip, K. and Wallsten, R.: Density theorems for sampling and interpolation in the BargmannFock space II. J. Reine Angew. Math. 429 (1992), p. 107-113. MR 93g:46026b

[50] Wojtaszczyk, P.: A mathematical introduction to wavelets. Cambridge University Press, 1997. MR 98j:42025

[51] Young, R.: An introduction to nonharmonic Fourier series. Academic Press, New York, 1980. MR 81 m:42027

Technical University of Denmark, Department of Mathematics, Building 303, 2800 LyNGBY, DENMARK

E-mail address: Ole.Christensen@mat.dtu.dk 